\title{
Appraisal of Maize Germplasm for Identification of Nutritionally Rich Nutraceutical Genotypes
}

\author{
R. KAUR and K. KAUR* \\ Department of Biochemistry, Punjab Agricultural University, Ludhiana, Punjab, India-141004
}

(Received 12 September 2015; Accepted 21 December 2015;

Communicated by J. Johnson)

\begin{abstract}
Thirty maize genotypes were characterized for their nutritional, antinutritional and nutraceutical traits for identification of elite germplasm rich in beneficial characteristics. Starch, proteins, lipids, tryptophan, lysine, $\beta$-carotene, condensed tannins, protease inhibitor, phytic acid, total phenols, flavonols, $o$-dihydroxy phenols and DPPH radical scavenging activity were determined in grains of all the genotypes. Correlation coefficient among the various parameters showed that lysine, total phenols, flavonols and $o$-dihydroxy phenols were negatively correlated with phytic acid content of the genotypes. This showed that the nutritional and the nutraceutical potency of genotypes, rich in lysine and phenolic compounds, is further propounded by a reduction of phytic acid content in them. On the basis of various constituents, the genotypes were divided into three groups. Group-A genotypes had high to moderate levels of both nutritional and nutraceutical traits. Group-B included those genotypes that exhibited high to moderate levels of either nutritional or nutraceutical traits. Group-C was constituted of genotypes that had lower levels of both nutritional and nutraceutical traits. Agglomerative hierarchical clustering showed that the D subcluster of MC-2 was chiefly comprised of genotypes that had high to moderate levels of both nutritional and nutraceutical traits. It may be concluded that CML134, CML266, TOO14601, LM11, CML264, CML321, SE563, LM10, LM18, LM14 and CML32 were nutritionally rich nutraceutical genotypes having low antinutrient potency.
\end{abstract}

Keywords: maize, nutritional aspects, antinutrients, nutraceuticals

\section{Introduction}

Maize, the poor man's nutria cereal, is an important crop of the family Poaceae. It ranks first in world production and acquires a well-deserved reputation in terms of human nutrition, animal feed and grain industry. By 2025, maize has been predicted to become the crop with the greatest production globally (Rosegrant et al. 2009). The golden grain accounts for about $15-56 \%$ of the total daily calories in the diets of people living in low and lower middle income countries, where animal protein is scarce and expensive and consequently unavailable to a vast sector of the population. Consequently, it is cultivated on nearly 184.2 million ha in the developing countries. In India, it occupies an area of 9.5

*Corresponding author; E-mail: kamaljit_pau@pau.edu; Phone: 09872971581 
million ha with an annual production of $\sim 23$ million tons (FAOSTAT 2014). Therefore, there is a strong need to exploit the maize genotypes for improved nutritional and nutraceutical characteristics so as to maintain a healthy living in the economically weaker sections of the world. With regard to this, several maize breeding programs, with special emphasis on grain nutritional quality are in progress (Jaradat and Goldstein 2013).

Seeds are the nutritious and edible parts of maize. The nutritional and economical value of maize kernel is primarily ascribable to its high starch content that contributes a significant proportion to the mature seed weight. However, the protein and oil content are also essential for human and animal nutrition. Nevertheless, the protein quality of maize gets restricted due to the deficiency of a few essential amino acids. In normal maize, bulk of the endosperm proteins is of prolamine (zeins) type. The high proportion of zeins in the endosperm is the primary reason for the poor protein quality as these are particularly deficient in lysine and tryptophan (Gutierrez-Rojas et al. 2008). Further, the nutritive value of maize also gets hampered by the presence of some antinutritional factors that reduce the utilization of nutrients. The levels of these biologically active components vary with the species and cultivar of plants (Zank 1991). Phytic acid, protease inhibitors and condensed tannins are the common antinutritional compounds that affect nutrient absorption in the body. Phytic acid acts as a powerful chelating agent that reduces the bioavailability of divalent cations by the formation of insoluble complexes (Lesteinne et al. 2005). Tannins have been reported to cause intestinal damage, interfere with iron absorption and produce carcinogenic effects (Chung et al. 1998). Protease inhibitors have the ability to inhibit the activity of proteolytic enzymes within the gastrointestinal tract of animals. Trypsin inhibitors have been implicated in reducing protein digestibility and causing pancreatic hypertrophy, by blocking the activity of trypsin (Smith et al. 1989).

In addition to nutritional traits, the nutraceutical quality of the food would suffice in the maintenance of a healthy living. These phytochemicals have health promoting, disease preventing and medicinal properties (Dureja et al. 2003). Nutraceutical substances maintain and potentiate the physiological or metabolic functions (Doyon and Labrecque 2008). Certain phytochemicals such as phenolics, anthocyanins and carotenoids have been reported for their antioxidant activity in maize (Zilic et al. 2012).

Although, biofortification of maize is a feasible way to improve its nutritional and nutraceutical quality, but it is more economical and liberal from public concerns to explore and utilize the genetic variations for these quality traits, in the available germplasm. Therefore, the objective of this study was to characterize the biochemical diversity present in maize germplasm so as to identify genotypes that are enriched with nutritional and nutraceutical constituents, but manage lower levels of antinutritional factors. This would help in the development of superior cultivars as well as promoting their consumption for a healthy, yet economical living. 


\section{Materials and Methods}

Thirty maize genotypes, having different genetic backgrounds (Table S1*) were grown under same environmental conditions and harvested in the year 2013, from the experimental area of the Department of Plant Breeding and Genetics, Punjab Agricultural University, Ludhiana, India.

Determination of starch, soluble proteins, tryptophan, lysine, lipids and $\beta$-carotene contents

The sugar free residue, obtained with $70 \%$ ethanol, was dried at $60{ }^{\circ} \mathrm{C}$ and used for the estimation of starch (Yoshida et al. 1976). Total soluble proteins were extracted with $0.1 \mathrm{~N} \mathrm{NaOH}$ and estimated (Lowry et al. 1951). The lipid content was determined by the method of Folch et al. (1957). Tryptophan and lysine were extracted and estimated by the procedure described by Nurit et al. (2009). $\beta$-Carotene was extracted and estimated by the method of AACC (1995).

\section{Determination of condensed tannins, protease inhibitor and phytic acid content}

Condensed tannins were extracted with methanol and estimated using vanillin reagent (Price et al. 1978). Protease inhibitor was extracted and estimated by the method of Hajela et al. (1999). Phytic acid was extracted (Zemel and Shelef 1982) and organic phosphorus was estimated by the method of Rouser et al. (1974).

\section{Determination of phenolic compounds and DPPH radical scavenging activity}

The powdered seeds were refluxed with $80 \%$ aqueous methanol. The refluxed material after filtration was used for the estimation of total phenols (Swain and Hillis 1959), o-dihydroxy phenols (Nair and Vaidyanathan 1964) and flavonols (Balaba et al. 1974). DPPH radical scavenging activity was extracted and estimated from the powdered seeds with methanol. For estimation, $0.1 \mathrm{mM} \mathrm{DPPH}$ was added to $1 \mathrm{ml}$ of supernatant, kept in the dark for $30 \mathrm{~min}$ and absorbance was read at $515 \mathrm{~nm}$ (Yamaguchi et al. 1998).

\section{Data analysis}

One-way analysis of variance followed by post-hoc analysis was applied to determine the significant differences among the maize genotypes. Correlation coefficient among various constituents was determined in maize germplasm. By XLSTAT 2015 software, hierarchical agglomerative clustering was done with Pearson correlation coefficient using flexible linkage.

*Further details about the Electronic Supplementary Material (ESM) can be found at the end of the article. 


\section{Results}

\section{Status of nutritional constituents}

The average content of starch was observed to be $660.5 \mathrm{mg} \mathrm{g}^{-1}$ seeds (Table S2). Among the various genotypes studied, CM139, CM143, CM144, LM5, LM14, LM15, LM16, LM17, LM18, I155, SE527, SE568, SE569 and TOO14601 were observed to be rich sources of starch (Table S2). Protein content varied from $7.1 \%$ to $13.3 \%$ with an average value of $10.1 \%$. Among the various genotypes, SE563 was observed to have the lowest protein content (Table S2). On the contrary, the highest content of soluble proteins was observed in LM10, CM139, CM140, CM143, LM5, LM14, LM15, LM16 and I168 genotypes (Table S2). Our results further showed that the tryptophan content varied from 0.005 to $0.048 \%$ among the genotypes, with LM17, LM18 and I168 showing the highest content, while SE527, I137 and CM144 showing the lowest content (Table S2). Lysine content was observed to be high in CML134, CML321, CML264, CML266, TOO14601 and CM143 genotypes (Table S2). The lipid content of the maize genotypes varied from $0.96-4.66 \%$ (Table S2). The highest content of lipids was observed in LM18, I155, LM14, I137 and SE563 genotypes. $\beta$-Carotene content varied from $0.56-8.33 \mathrm{ppm}$ with a mean value of $4.62 \mathrm{ppm}$ (Table S2). CML134 genotype was found to have the lowest content of $\beta$-carotene while highest content was observed in LM19 (Table S2). In addition, LM11, LM18, LM20, I164, SE569 and TOO14601 also showed high levels of $\beta$-carotene in their grains.

\section{Status of antinutritional constituents}

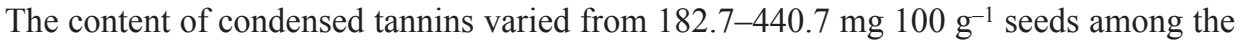
maize genotypes. Mean value of condensed tannins was found to be $299.1 \mathrm{mg} 100 \mathrm{~g}^{-1}$ (Table S3). LM15 and LM12 were found to have the lowest content of condensed tannins. However, LM5, LM10, LM14, LM17, LM18, LM19, I155, I137, I168, CML32, SE569, CML321, CML134 and CML 266 genotypes were rich in the content of condensed tannins (Table S3). Their condensed tannins content was observed to be $\geq 300 \mathrm{mg} / 100 \mathrm{~g}$. Our results further showed that a few genotypes, namely LM11, I137, CML32 and CML134 had very low protease inhibitor activity with CML266 exhibiting the lowest activity of 45.8 units $\mathrm{g}^{-1}$ seeds. On the other hand, the protease inhibitor activity was observed to be more than 200 units $\mathrm{g}^{-1}$ in CM143, CM144, LM12 and LM17 genotypes (Table S3). Maize genotypes were found to have a significant variation in the content of phytic acid as it ranged from as low as $2.16 \mathrm{mg} \mathrm{g}^{-1}$ seeds to as high as $8.47 \mathrm{mg} \mathrm{g}^{-1}$ seeds (Table S3). A few genotypes, e.g. LM11, I168, TOO14601, CML32, CML264, CML321, CML134 and CML266 were observed to have very low phytic acid content (i.e. less than $3.0 \mathrm{mg}$ $\mathrm{g}^{-1}$ seeds) (Table $\mathrm{S} 3$ ).

\section{Status of nutraceutical constituents}

It was observed that the total phenolic content varied from $58.13-153.33 \mathrm{mg} 100 \mathrm{~g}^{-1}$ seeds with a mean value of $90.65 \mathrm{mg} 100 \mathrm{~g}^{-1}$ seeds (Table S4). LM5 and LM10 were 
found to have the lowest content while CML264 and CML321 were observed to have the highest content of total phenols in the grains (Table S4). In general, all the CML genotypes, viz. CML264, CML321, CML134, CML266 and CML32 along with TOO1461, CM140, LM12, LM14, SE568 and SE569 had high total phenolic content (Table S4).

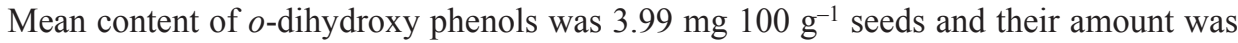
found to vary in the range of 2.09-6.53 mg $100 \mathrm{~g}^{-1}$ seeds (Table S4). LM13, LM5, I155 and I137 genotypes had the lowest content of $o$-dihydroxy phenols, while its highest content was observed in LM11 genotype. Other genotypes showing relatively high content of o-dihydroxy phenols were LM12, LM18, CML134, CML266 and CML321 (Table S4). Flavonols were found to lie in the range of $13.83-53.57 \mathrm{mg} 100 \mathrm{~g} \mathrm{~g}^{-1}$ seeds with an average

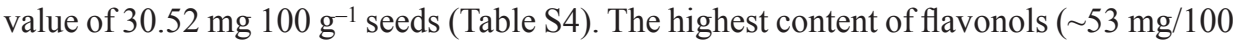
g) was observed in CML264 and CML321 genotypes. The genotypes, viz. CML134, CML266, TOO14601, LM12, LM13, LM18, I155, I137, I164, SE568 and SE569 showed their flavonol content above $30 \mathrm{mg} 100 \mathrm{~g}^{-1}$ (Table S4). DPPH free radical scavenging activity varied from 38.53-63.33\% among the genotypes (Table S4). In general, almost all maize genotypes showed high DPPH radical scavenging activity (Table S4).

Correlation coefficient among nutritional, antinutritional and nutraceutical constituents of maize genotypes

Correlation coefficient among nutritional, nutraceutical and antinutritional components of maize genotypes was studied (Table 1). Phytic acid content was observed to be negatively correlated with flavonols $(\mathrm{r}=-0.433), o$-dihydroxy phenols $(\mathrm{r}=-0.449)$, total phenols $(\mathrm{r}=-0.496)$ and lysine $(\mathrm{r}=-0.578)$. Total phenolic content was observed to be positively correlated with flavonols and $o$-dihydroxy phenols $(\mathrm{r}=0.749$ and 0.398 , respectively). However, it showed negative correlation with $\beta$-carotene $(r=-0.638)$. $\beta$-Carotene also showed negative correlation with flavonols $(\mathrm{r}=-0.458)$ but positive correlation with phytic acid $(\mathrm{r}=0.580)$. Lysine was found to be positively correlated with flavonols and total phenols ( $\mathrm{r}=0.587$ and 0.681 , respectively) but showed negative correlation with $\beta$-carotene $(\mathrm{r}=-0.652)$. Starch was found to be positively correlated with total proteins $(\mathrm{r}=0.379)$. However, lipid content was found to be negatively correlated with total proteins. Tryptophan was observed to be positively correlated with condensed tannins (Table 1).

\section{Agglomerative hierarchical clustering}

Agglomerative hierarchical clustering using Pearson's correlation coefficient with flexible linkage divided the genotypes into two main clusters: MC-1 and MC-2. MC-1 was subdivided into two subclusters A and B while MC-2 was divided into $\mathrm{C}$ and $\mathrm{D}$ sub clusters (Fig. 1). It was observed that $\mathrm{MC}-1$ and $\mathrm{C}$ subcluster of $\mathrm{MC}-2$ was constituted mostly of those genotypes that exhibited high to moderate levels of either nutritional or nutraceutical traits, while the D subcluster of MC-2 was comprised of genotypes having high to moderate levels of both nutritional and nutraceutical traits. There were seven geno- 


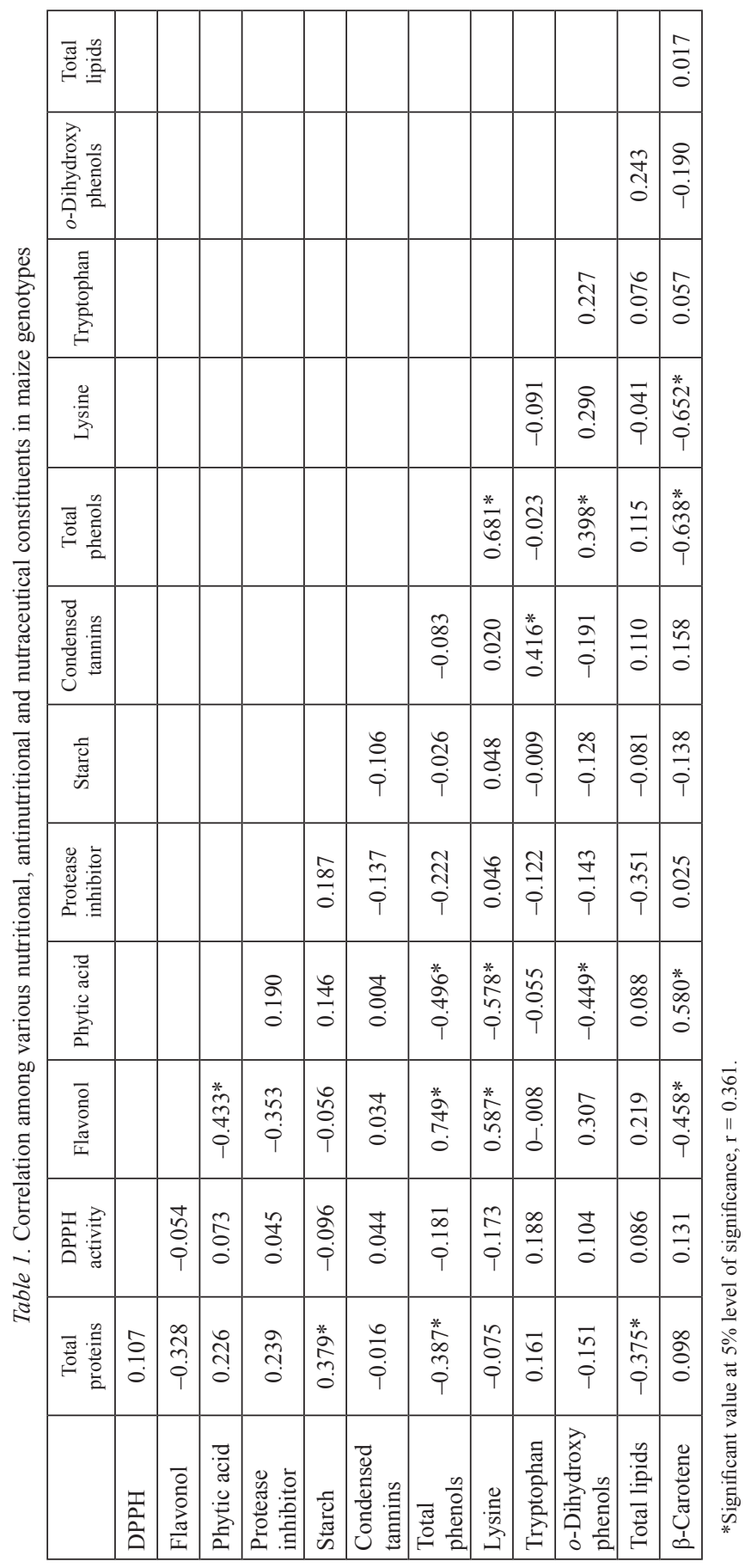


types that had lower levels of both nutritional or nutraceutical traits (Fig. 1). These were observed to be scattered throughout the dendrogram with three genotypes in MC-1 and four in MC-2 (Fig. 1).

\section{Dendrogram}

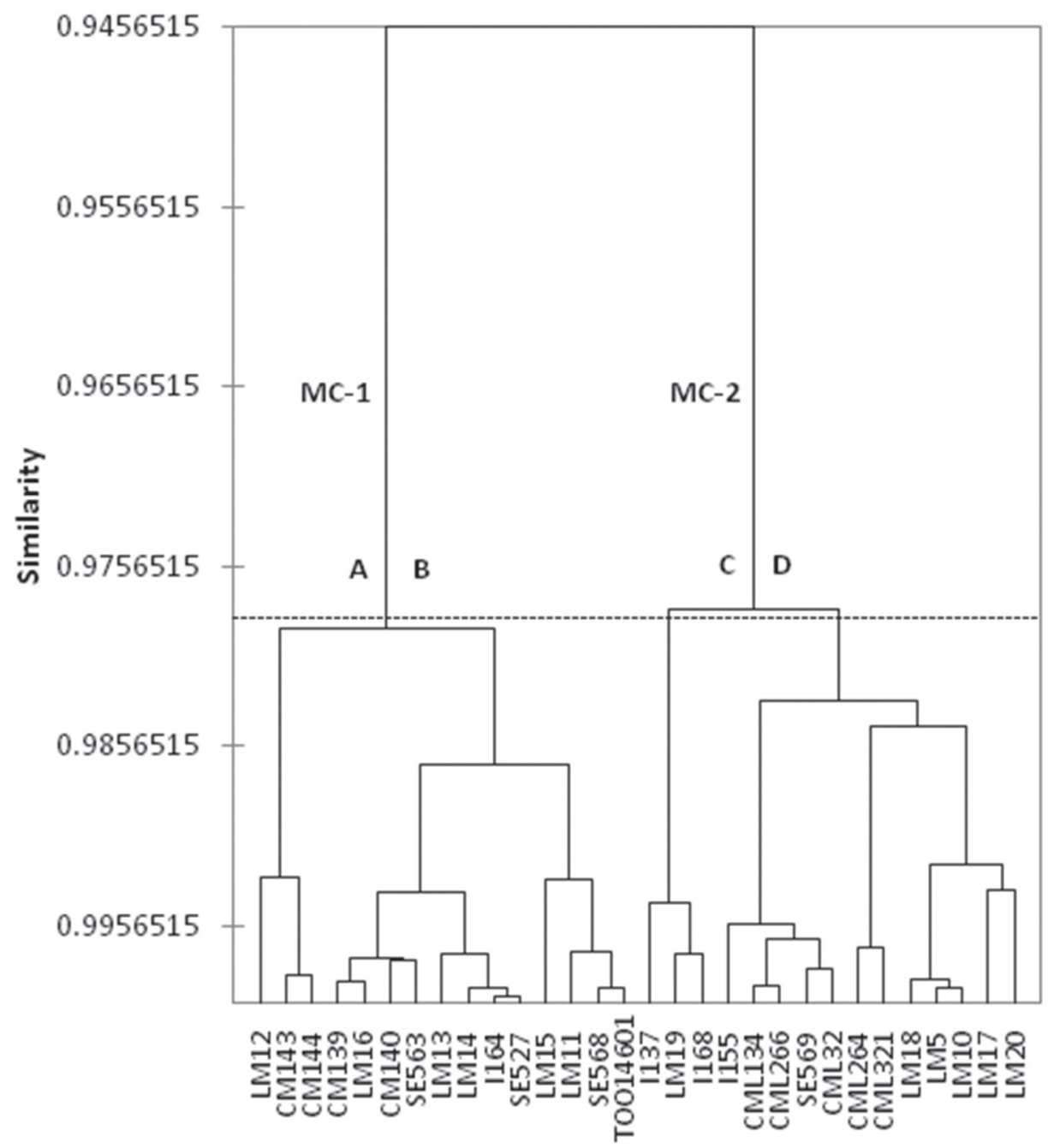

Figure 1. Dendrogram of maize genotypes obtained with Pearson's correlation coefficient using flexible linkage MC-1, major cluster-1; MC-2, major cluster-2; A and B, subclusters of MC-1; C and D, subclusters of MC-2 


\section{Discussion}

Maize grain has been reported to be a rich source of starch as compared to other major food crops such as wheat and rice (Chander et al. 2008). Our results are consistent with those given by Jaradat and Goldstein (2013). The enlisted genotypes, rich in starch content (Table S2), gave them an upper hand in terms of nutritional value. Protein is another important chemical constituent of grains. Literature, however, reported that protein content of maize lie in the range of $9-11 \%$ (Sofi et al. 2009). It has been reported that $50-70 \%$ of the endosperm proteins are the zein proteins which are deficient in tryptophan and lysine and consequently lead to poor nutritional quality (Gibbon and Larkins 2005). Our results of tryptophan content showed that LM17, LM18 and I168 genotypes are nutritionally enriched with the amino acid that is mainly low in maize (Table S2). On the other hand, SE527, I137 and CM144 genotypes might be regarded as less nutritional in terms of their low tryptophan content (Table S2). The genotypes, CML134, CML321, CML264, CML266, TOO14601 and CM143 have a better nutritional status than others in terms of their lysine content. These results further showed that I168 and CM 143 are tryptophan and lysine rich, high protein grains, respectively (Table S2). The nutritional importance of maize grain is also attributed to the oil stored in the embryo. From the results, it was inferred that LM18, I155, LM14, I137 and SE563 are nutritionally better with respect to their lipid content (Table S2). $\beta$-Carotene plays a vital role in the human body because of its pro-vitamin A activity (Chen et al. 1993). It was inferred that LM11, LM18, LM19, LM20, I164, SE569 and TOO14601were highly nutritious with respect to their $\beta$-carotene content (Table S2).

However, the nutritive value of food depends upon the status of antinutritional compounds in them. Tannins have been reported to interact with both enzyme and non-enzyme proteins to form tannin-protein complexes that lead to the inactivation of digestive enzymes thereby reducing food digestibility (Khandelwal et al. 2010). Tannins, being resistant to degradation, remain in the digestive tract for long periods. Therefore, the high tannin content of enlisted genotypes proves to be a negative trait of these genotypes (Table S3). Protease inhibitors have been reported to form stable complexes with proteolytic enzymes, thus reducing the hydrolysis of dietary proteins leading to decreased amino acid absorption and de novo protein synthesis (Roy et al. 2010). The lower protease inhibitor activity of CML266, LM11, I137, CML32 and CML134 could be attributed as a constructive trait for making these genotypes superior than others (Table S3). On the other hand, the high protease inhibitor activity in CM143, CM144, LM12 and LM17 genotypes could make them less nutritional (Table S3). Phytic acid is the principal storage form of phosphorus in plant tissues (Doria et al. 2009). During germination, it serves as a major source of phosphorus for the growing seedlings. Phytic acid has been reported to have both nutritional as well as antinutritional effects. It has been reported as an anticarcinogenic agent as well as an important therapeutic against diabetes mellitus, atherosclerosis and coronary heart diseases (Kumar et al. 2010). However, it also acts as a strong chelator of divalent cations like calcium, magnesium, iron and zinc, and thus, contributes to mineral deficiency by reducing their bioavailability (Lesteinne et al. 2005). 
It also forms insoluble complexes by binding starch and protein molecules, thereby reducing their bioavailability in humans (Phillippy 2003). It could be inferred that LM11, CML32, CML134 and CML266 have better nutritional quality as they had very low levels of both the antinutritional constituents, viz. protease inhibitor activity and phytic acid content (Table S3), making these genotypes beneficial for human consumption.

Phenols have also been termed as antinutritional because of their tendency to bind positively charged proteins and minerals in foods and reduce their digestibility (Gilani et al. 2005). However, these antinutritional traits have been trounced by their ability to act as reducing agents, hydrogen donors and chelators of metal ions (Decker 1995) as well as their ability to act as inhibitors of low density lipoproteins, cholesterol oxidation and DNA breakage (Shahidi 2004). From our results, it could be inferred that CML264, CML321, CML134, CML266 and CML32 along with TOO1461, CM140, LM12, LM14, SE568 and SE569 can be regarded as total phenol rich nutraceutical genotypes. The higher content of $o$-dihydroxy phenols in a few genotypes, viz., LM11, LM12, LM18, CML134, CML266 and CML321 also appeared to make them nutraceutically beneficial (Table S4). Flavonols have been reported to exert beneficial effects on the central nervous system by protecting neurons against stress-induced injury through suppression of neuroinflammation (Letenneur et al. 2007). In concert with total phenol content, CML264, CML321, CML134, CML266, TOO14601, LM12, SE568 and SE569 genotypes also showed high flavonol content which thus potentiates their nutraceutical efficiency. In addition, LM13, LM18, I155, I137, I164 genotypes also appeared to be nutraceutically beneficial as they have high antioxidant properties of flavonols, which can strongly inhibit the reactive oxygen species (Table S4). The nutraceutical status of maize is also attributed to the DPPH free radical scavenging activity that showed significant levels among all the genotypes (Table S4). The high antioxidant activity of maize grain has been reported to be associated with phenolic compounds present in the grain (Fardet et al. 2008). In fact, the antioxidant ability of the grains has been reported to be positively correlated with phenolics content (Verma et al. 2008). However, our results did not show any such positive correlation between total phenols and DPPH free radical scavenging activity (Table S4).

From the results of correlation coefficient (Table 1), it may be concluded that the characteristics of high lysine, total phenols, flavonols and $o$-dihydroxy phenols tend to be in concert with low phytic acid content in maize. Thus the nutritional and the nutraceutical potency of maize genotypes, enriched with lysine and phenolic compounds, would increase due to a reduction in their phytic acid content. This showed that the genotypes, e.g., LM18, CML134, CML264, CML266 and TOO14601 having high contents of lysine, total phenols, flavonols and $o$-dihydroxy phenols would be nutritionally and nutraceutically more efficient because of their low phytic acid content (Tables S3 and S4).

On the basis of the levels of the various constituents, the genotypes were divided into three groups. Group-A was primarily constituted of those genotypes which showed high to moderate levels of both the nutritional and nutraceutical traits. Group-B contained those genotypes that exhibited high to moderate levels of either nutritional or nutraceutical traits. The third Group-C constituted of those genotypes which had lower levels of 
both nutritional and nutraceutical traits. The results thus showed that the genotypes viz., CML134, CML266, TOO14601, LM11, CML264, CML321, SE563, LM10, LM18, LM14 and CML32 were present in Group-A. The Group-B was comprised of CM143, SE568, LM5, I155, CM139, CM144, LM13, LM15, I137, I168, LM12 and 164 genotypes. CM140, SE527, LM16, LM17, LM19, LM20 and SE569 constituted the Group-C genotypes. Agglomerative hierarchical clustering using Pearson's correlation coefficient with flexible linkage clustered the genotypes into certain specific clusters (Fig. 1). It was observed that $\mathrm{MC}-1$ and $\mathrm{C}$ subcluster of $\mathrm{MC}-2$ was constituted mostly of Group-B genotypes, while the D subcluster of MC-2 was comprised of Group-A genotypes, having high to moderate levels of both nutritional and nutraceutical traits (Fig. 1).

It may be concluded that the genotypes, viz. CML134, CML266, TOO14601, LM11, CML264, CML321, SE563, LM10, LM18, LM14 and CML32 could be ranked in the best category on the basis of their nutritional and nutraceutical properties. These genotypes thus provide elite breeding stocks for the enrichment of nutritionally and nutraceutically important constituents.

\section{Acknowledgement}

The authors are grateful to Dr. M.S. Grewal, Dept. of Plant Breeding and Genetics, Punjab Agricultural University, Ludhiana for providing maize germplasm.

\section{References}

AACC 1995. Approved Methods of the American Association of Cereal Chemists (AACC). Am. Assoc. Cereal Chem. St. Paul, MN, USA pp. 14-50.

Balaba, S.I., Zake, A.Y., Elshamy, A.M. 1974. Total flavonoids and rutin content of the different organs of Japonica L. J. Assoc. of Official Anal. Chemists 57:752-755.

Chander, S.Y., Meng, Y., Zhang, J., Yan, J.B., Li, J. 2008. Comparison of nutritional traits variability in selected eighty seven inbreds from Chinese maize (Zea mays L.) germplasm. J. Agric. Food Chem. 56:6506-6511.

Chen, B.H., Chuang, J.R., Lin, J.H., Chiu, C.P. 1993. Quantification of pro-vitamin A compounds in Chinese vegetables by high-performance liquid chromatography. J. Food Protection 56:51-54.

Chung, K.T., Wong, T.Y., Wei, C.I., Huang, Y.W., Lin, Y. 1998. Tannins and human health: A review. Critical Reviews in Food Sci. and Nutrition 38:421-464.

Decker, E.A. 1995. The phenolic, conjugated linoleic acid, carnosine and pyrroloquinoline quinine as nonessential dietary antioxidants. Nutrition Reviews 53:49-58.

Doria, E., Galleschi, L., Calucci, L., Pinzino, C., Pilu, R., Cassani, C., Nielsen, E. 2009. Phytic acid prevents oxidative stress in seeds: evidence from a maize (Zea mays L.) low phytic acid mutant. J. Exp. Bot. 60:967-978.

Doyon, M., Labrecque, J. 2008. Functional foods: a conceptual definition. British Food J. 110:1133-1149.

Dureja, H., Kaushik, D., Kumar, V. 2003. Developments in nutraceuticals. Indian J. Pharmacol. 35:363-372.

FAOSTAT 2014. Statistical databases and data sets of the food and agriculture organization of the United Nations (http://faostat.fao.org/default.aspx)

Fardet, A., Rock, E., Rémésy, C. 2008. Is the in vitro antioxidant potential of whole-grain cereals and cereal products well reflected in vivo? J. Cereal Sci. 48:258-276.

Folch, J., Lees, M., Sloane-Stanley, G.H. 1957. A simple method for isolation and purification of total lipids from animal tissues. J. Biol. Chem. 226:497-506. 
Gibbon, B.C., Larkins, B.A. 2005. Molecular genetics approaches to developing quality protein maize. Trends in Genetics 21:227-233.

Gilani, A.H., Bashir, S., Janbaz, K.H., Shah, A.J. 2005. Presence of cholinergic and calcium channel blocking activities explains the traditional use of Hibiscus rosasinensis in constipation and diarrhea. J. Ethnopharmacology 102:94-289.

Gutierrez-Rojas, A., Scott, M.P., Levy, O.R., Menz, M., Betran, J. 2008. Phenotypic characterization of quality protein maize endosperm modification and amino acid contents in a segregating recombinant inbred population. Crop Sci. 48:1714-1722.

Hajela, N., Pande, A.H., Sharma, S., Rao, D.N., Hajela, K. 1999. Studies on a double headed protease inhibitors from Phaselous mungo. J. Plant Biotechnol. 8:57-60.

Jaradat, A.A., Goldstein, W. 2013. Diversity of maize kernels from a breeding program for protein quality: physical, biochemical, nutrient and color traits. Crop Sci. 53:956-976.

Khandelwal, S., Udipi, S.A., Ghugre, P. 2010. Polyphenols and tannins in Indian pulses: Effect of soaking, germination and pressure cooking. Food Res. Int. 43:526-530.

Kumar, V., Sinha, A.K., Makkar, H.P.S., Becker, K. 2010. Dietary roles of phytate and phytase in human nutrition: A review. Food Chem. 120:949-959.

Lesteinne, I., Rivier, C.M., Verniere, C.I., Rochette, I., Treche, S. 2005. The effects of soaking of whole, dehulled and ground millet and soybean seeds on phytate degradation and Phy/Fe and Phy/Zn molar ratios. Int. J. Food Sci. and Technol. 40:391-399.

Letenneur, L., Proust-Lima, C., Le-Gouge, A., Dartigues, J.F., Barberger-Gateau, P. 2007. Flavonoid intake and cognitive decline over a 10-year period. Am. J. Epidemiology 165:1364-1371.

Lowry, O.H., Rosebrough, N.J., Farr, A.L., Randall, R.J. 1951. Protein measurement with the folin phenol reagent. J. Biol. Chem. 193:265-275.

Nair, P.M., Vaidyanathan, C.S. 1964. A colorimetric method for the determination of pyrocatechol and related compounds. Anal. Biochem. 7:315-321.

Nurit, E., Tiessen, A., Pixley, K.V., Rojas, N.P. 2009. Reliable and inexpensive colorimetric method for determining protein-bound tryptophan in maize kernels. J. of Agric. and Food Chem. 57:7233-7238.

Phillippy, B.Q. 2003. Inositol phosphates in foods. Advances in Food and Nutrition Res. 45:1-60.

Price, M.L., Van Scooc, S., Butler, L.G. 1978. A critical evaluation of the vanillin reaction as an assay for tannins and sorghum grain. J. of Agric. and Food Chem. 26:12-14.

Rosegrant, M.R., Ringler, C., Sulser, T.B., Ewing, M., Palazzo, A., Zhu, T. 2009. Agriculture and food security under global change: Prospects for 2025/2050. International Food Policy Research Institute. Washington D.C., USA.

Rouser, G., Fleisher, S., Yamamoto, A. 1974. Two-dimensional thin layer chromatographic separation of polar lipids and determination of phospholipids by phosphorous analysis of spots. Lipids 5:494-496.

Roy, A.F., Boye, J.I., Simpson, B.K. 2010. A review bioactive proteins and peptides in pulse crops: pea, chickpea and lentil. Food Res. Int. 43:433-442.

Shahidi, F. 2004. Quality characteristics of edible oils. In: Shahidi, F., Spanier, A.M., Ho, C.T., Braggins, T. (eds.), Quality of Fresh and Processed Foods. Kluwer Academic/Plenum Publishers. New York, USA. pp. 239-249.

Sofi, P.A., Wani, S.A., Rather, A.G., Wani, S.H. 2009. Review article: quality protein maize (QPM): Genetic manipulation for the nutritional fortification of maize. J. Plant Breeding and Crop Sci. 1:244-253.

Smith, J.C., Wilson, F.D., Allan, P.V., Berry, D.L.1989. Hypertrophy and hyperplasia of the rat pancreas produced by the short-term dietary administration of soya derived protein and soybean trypsin inhibitor. J. Appl. Toxicol. 9:175-179.

Swain, T., Hills, E. 1959. The phenolic constituents of Prunus domestica. The quantitative analysis of phenolic constituents. J. of the Sci. of Food and Agric. 10:63-68.

Verma, B., Hucl, P., Chibbar, R.N. 2008. Phenolic content and antioxidant properties of bran in 51 wheat cultivar. Cereal Chem. 85:544-549.

Yamaguchi, T., Takamura, H., Matoba, T., Terao, J. 1998. HPLC method for evaluation of the free radicalscavenging activity of foods by using 1, 1,-diphenyl-2-picrylhydrazyl. Biosci., Biotechnol. and Biochem. 62:1201-1204. 
Yoshida, S., Forno, D., Cock, J., Gomez, K. 1976. Determination of sugar and starch in plant tissue. In: Yoshida, S. (ed.), Laboratory Manual for Physiological Studies of Rice. The International Rice Research Institute. Los Banos, The Philippines. pp. 46-49.

Zank, H.M. 1991. Chasing the enzymes for secondary metabolism: Plant cell cultures as a point of goal. Phytochem. 309:3861-3863.

Zemel, M.B., Shelef, L.A. 1982. Phytic acid hydrolysis with soluble zinc and iron in whole wheat bread as affected by calcium containing additives. J. Food Sci. 47:535-537.

Zilic, S., Serpen, A., Akillioglu, G., Gokmen, V., Vancetovic, J. 2012. Phenolic compounds, carotenoids, anthocyanins and antioxidant capacity of colored maize (Zea mays L.) kernels. J. Agric. and Food Chem. 60:1224-1231.

\section{Electronic Supplementary Material (ESM)}

Electronic Supplementary Material (ESM) associated with this article can be found at the website of CRC at http://www.akademiai.com/content/120427/

Electronic Supplementary Table S1. Pedigree of the maize genotypes taken for investigation

Electronic Supplementary Table S2. Distribution of starch, soluble proteins, tryptophan, lysine,total lipids and $\beta$-carotene in maize genotypes

Electronic Supplementary Table S3. Distribution of condensed tannins, protease inhibitor activity and phytic acid content in maize genotypes

Electronic Supplementary Table S4. Distribution of total phenols, o-dihydroxy phenols, flavonols and DPPH free radical scavenging activity in maize genotypes 